\title{
Eficacia de un modelo integral de dieta muy baja en calorías con reemplazo de comida y liraglutida en reducción de peso en pacientes con obesidad
}

\author{
Effectiveness of a comprehensive model of a very low-calorie diet \\ with meal replacement and liraglutide for weight reduction in patients \\ with obesity
}

Rosero O,1 Pantoja D, ${ }^{2}$ Pabón $N,{ }^{2}$ Ibarra J. ${ }^{3}$

${ }^{1}$ Médico endocrinólogo. Osteollanos Villavicencio, Balance
Villavicencio. Villavicencio, Colombia.
${ }^{2}$ Médico endocrinólogo. CENDO Pasto, Balance Pasto. Pasto,
Colombia.
${ }^{3}$ Médico epidemiólogo. CENDO Pasto, Balance Pasto. Pasto,
Colombia.

Autor de correspondencia: Óscar Rosero

Correo electrónico: oscarrosero@yahoo.com

Fecha de recepción: 31/08/2020

Fecha de aceptación: 12/02/2021

\section{Resumen}

Introducción: la prevalencia de la obesidad en los últimos años ha aumentado a nivel mundial, convirtiéndose en un problema de salud pública. Lograr una disminución de peso se ha vuelto un reto tanto para el personal de salud como para los pacientes: una disminución del $5 \%$ al $10 \%$ del peso en pacientes con obesidad o sobrepeso impacta positivamente en la morbimortalidad. En el presente estudio se describen los resultados de un programa de intervención terapéutica dirigido a lograr la disminución de peso en una población con obesidad.

Metodología: estudio descriptivo, retrospectivo de un grupo de 63 pacientes que ingresaron a un programa integral de restricción calórica controlada con el uso de liraglutida, dosis titulada, de 3 meses de duración, en la ciudad de Pasto, Nariño, desde abril de 2018 hasta diciembre de 2019.

Resultados: el 77,7 \% de pacientes fueron mujeres y el $22,3 \%$, hombres; con una media de edad de 43,4 años. En promedio, se logró una disminución del 11,85 \% del peso inicial, una pérdida de grasa corporal de 11,67 \% y una disminución en el perímetro abdominal de 13,2 cm; no hubo cambios esta- dísticamente significativos en la masa magra corporal ni en el metabolismo basal.

Conclusión: la metodología planteada, de un modelo integral multidisciplinario, de restricción calórica, con el uso de liraglutida puede ser una estrategia efectiva en la pérdida de peso significativa a corto plazo en pacientes con obesidad, sin disminución estadísticamente significativa de la masa magra ni de la tasa metabólica en reposo.

Palabras clave: obesidad, dieta, peso corporal, pérdida de peso, estilo de vida.

\section{Abstract}

Introduction: In recent years, the prevalence of obesity has been growing worldwide, becoming a public health problem. Achieving a decrease in weight has become a challenge for both healthcare personnel as well as patients: a decrease of $5 \%$ to $10 \%$ in weight in obese or overweight patients has a positive impact on morbidity and mortality. This study describes the results of a therapeutic intervention program aimed at achieving weight loss in an obese population.

Methodology: a descriptive, retrospective study of a group of 63 patients who entered a comprehensive program of controlled caloric restriction, with the use of liraglutide titled, 3-month duration called The Balance Method, in the city of Pasto, Nariño, from April 2018 to December 2019.

Results: $77.7 \%$ were women and $22.3 \%$, men, with a mean age of 43.4 years. On average, there was a decrease of $11.85 \%$ in body, a loss of body fat of $11.67 \%$ and a decrease in abdominal circumference of $13.2 \mathrm{~cm}$; there were no statistically significant changes in lean body mass or basal metabolism.

Conclusion: The proposed methodology of a comprehensive multidisciplinary caloric restriction model with the use of 
liraglutida may be an effective strategy for significant shortterm weight loss in obese patients, without statistically significant decrease in lean mass or muscle mass. your resting metabolic rate.

Keywords: Obesity; Diet; Body Weight; Weight Loss; Lifestyle.

\section{Introducción}

La obesidad es una enfermedad compleja y multifactorial. La prevalencia mundial de sobrepeso y obesidad se ha duplicado desde 1980 hasta el punto de que casi un tercio de la población mundial ahora está clasificada como con alguna de estas alteraciones (1). Representa aproximadamente 4 millones de muertes al año en todo el mundo y más de dos tercios de las muertes secundarias a la obesidad están relacionadas con enfermedades cardiovasculares (1).

Una disminución de peso del $5 \%$ al $10 \%$ en personas con sobrepeso y obesidad mejora varios factores de riesgo para enfermedad cardiovascular (ECV), entre los que se encuentran glucosa elevada, tensión arterial elevada e hipertrigliceridemia (2). Una pérdida de peso de esta magnitud se puede lograr con programas que modifiquen los estilos de vida de manera integral, combinando dieta, actividad física y terapia comportamental (2).

Actualmente se plantean diferentes modelos de dieta que han demostrado eficacia en la disminución de peso, como la dieta muy baja en calorías (VLCD), dieta baja en carbohidratos, dieta baja en grasas, dieta mediterránea, dieta con bajo índice glucémico y dietas con alto contenido proteico $(3,4)$.

Una estrategia segura y que facilita la pérdida de peso es el cambio de una de las comidas por un suplemento proteico fortificado con vitaminas dentro del plan nutricional, especialmente en los planes de VLCD, lo que mejora la adherencia del paciente a un régimen de pérdida de peso (5).

Adicionalmente, estudios previos han demostrado que, con técnicas motivacionales, controles periódicos y patrones de alimentación fáciles de seguir, la efectividad en la pérdida de peso será mayor (2). El tratamiento farmacológico ofrece otra opción adicional y complementaria a los programas de disminución de peso (6).

Ante el panorama mundial que muestra a la obesidad como una epidemia, su elevada morbimortalidad y la dificultad encontrada para lograr metas con respecto a la disminución de peso, se plantea un programa integral multifase, que integra el componente nutricional, el seguimiento a través del análisis de la composición corporal, el componente farmacológico (liraglutida) y el componente conductual (coaching) con disponibilidad de asesoramiento virtual por chat en forma permanente.

\section{Metodología}

Se recolectaron datos de manera retrospectiva de un grupo de 63 pacientes que ingresaron a un programa estructurado de control de peso, que consistía en 3 fases: la primera fue de 28 días con un plan de alimentación hipocalórico, con la ingesta de $50 \mathrm{~g}$ /día de carbohidratos y reemplazo de la cena con un suplemento a base de proteína de soya de $10 \mathrm{~g}$ por porción; la segunda fase fue de 28 días de duración y consistió en un modelo de dieta baja en calorías con un incremento gradual de aporte calórico y el mismo reemplazo de la cena; y la tercera fase tuvo un modelo de dieta bajo en calorías hasta llegar al final con el aporte calórico basal más las correcciones por actividad física de cada paciente, sin reemplazo de cena.

Se utilizó liraglutida para el control del apetito a dosis tituladas por el médico según la tolerancia y sensación de hambre durante los 3 meses de seguimiento, con una dosis de inicio en 0,6 mg/día por vía subcutánea hasta una dosis de 3,0 mg/día, ajustada según el reporte subjetivo de sensación de hambre del paciente. Los pacientes contaron con un entrenador físico para clases individuales de actividad física de resistencia durante la duración del proceso, a razón de 2 clases por semana de 30 minutos de duración e intensidad moderada; y se realizó una sesión de psicoterapia por personal entrenado. Desde el inicio los pacientes contaron con el acompañamiento vía virtual permanente para consultas y consejería sobre alimentación saludable y estilos de vida. Los pacientes fueron controlados por el médico de forma presencial cada semana con la toma de signos vitales y el análisis de la composición corporal desde abril de 2018 hasta diciembre de 2019 en la ciudad de Pasto, Colombia. Se tomaron los datos del ingreso al programa y a los 3 meses, luego de terminarse. Entre las variables recolectadas se encuentran la edad, sexo, variables antropométricas (peso, talla, índice de masa corporal [IMC], porcentaje de grasa corporal, porcentaje de músculo corporal, perímetro abdominal), metabolismo basal en reposo (calculado por el equipo de composición corporal Inbody-Harris Benedict) y dosis máxima de liraglutida utilizada. Las variables antropométricas (peso, porcentaje de grasa, porcentaje de músculo, metabolismo basal) se recolectaron a través de bioimpendaciometría de 8 puntos en el equipo Inbody $370 \AA$ para la composición corporal; la talla y el perímetro abdominal fueron tomados con cinta métrica por personal capacitado en el consultorio, el IMC se calculó a partir del peso y talla, y la dosis máxima de liraglutida se tomó de los datos de cada control.

Los datos se analizaron con el paquete estadístico de RStudio (7). Se realizó el análisis descriptivo para las variables cualitativas y cuantitativas, y el cálculo del porcentaje de cambio en cada variable; y se compararon los datos iniciales con los finales por medio de la prueba t de Student para variables dependientes con un intervalo de confianza (IC) del $95 \%$.

Por tratarse de un estudio descriptivo retrospectivo mediante la utilización de información ya existente, sin datos de carácter personal, no preciso sometimiento a comité de ética.

\section{Resultados}

De los 63 pacientes que ingresaron al programa durante este período, con características descritas en la Tabla 1 des- 
pués de la intervención, en promedio se logró una disminución del 11,85 \% en el peso inicial.

Se observó una pérdida de grasa corporal promedio del 11,67 \% y se evidenció una disminución en el perímetro abdominal de 13,2 cm (Tabla 2). Las anteriores variables tuvieron una reducción estadísticamente significativa comparadas con las mediciones iniciales $(p=0,000)$. Con respecto al porcentaje de masa magra y tasa metabólica en reposo, hubo una leve disminución al finalizar el seguimiento, sin que esta fuera estadísticamente significativa ( $p=0,235$ y 0,296 , respectivamente) (Tabla 3)

En las Tablas 1 y 2 se puede evidenciar las diferencias por sexo para las variables iniciales y finales. Cabe resaltar la diferencia entre hombres y mujeres con respecto a la composición corporal: las mujeres presentaron un mayor porcentaje de grasa corporal y un menor porcentaje de músculo (42,3\% y $24,7 \%$, respectivamente), mientras que en los hombres presentaron una distribución de grasa y músculo similar al ingreso del programa $(36,8 \%$ y $34,8 \%$, respectivamente). También es importante observar la diferencia en el metabolismo basal entre hombres y mujeres, que en promedio es 353 calorías más elevado en los hombres (1694 calorías en los hombres y 1341 calorías en las mujeres).

\section{Discusión}

Nuestro grupo evaluó de forma retrospectiva los resultados en peso y otras variables metabólicas de un modelo de atención integral a corto plazo, en un grupo de pacientes con obesidad y sobrepeso. La media de pérdida de peso promedio fue $11,67 \%(9,68 \mathrm{~kg})$ en 3 meses. Nuestros resultados son similares a los reportados por los estudios clínicos de fase III para la molécula liraglutida (6), confirmando la eficacia de este tipo de intervención dietética acompañado de terapia farmacológica, en este caso liraglutida.

La reducción del perímetro abdominal promedio fue de $13,2 \mathrm{~cm}$, la medida universal de adiposidad visceral fue estadísticamente significativa, y este resultado es importante pues

Tabla 1. Variables antropométricas iniciales

\begin{tabular}{|c|c|c|c|c|}
\hline Variable & Media & DE & Mujeres & Hombres \\
\hline & & & \multicolumn{2}{|c|}{ Media (DE) } \\
\hline Edad (años) & 43,4 & 9,98 & $42,8(9,6)$ & $45,6(11,3)$ \\
\hline Peso (kg) & 82,4 & 12,49 & $78,2(8,05)$ & $97,3(14,14)$ \\
\hline $\mathrm{IMC}\left(\mathrm{kg} / \mathrm{m}^{2}\right)$ & 31,2 & 3,35 & $30,6(3,05)$ & $33,3(3,59)$ \\
\hline Porcentaje de grasa corporal & 41,06 & 5,3 & $42,3(5,11)$ & $36,8(3,54)$ \\
\hline Porcentaje de masa magra corporal & 27,02 & 5,4 & $24,7(2,67)$ & $34,8(5,39)$ \\
\hline Perímetro abdominal (cm) & 103 & 11,8 & $99,7(9,99)$ & $114,9(9,98)$ \\
\hline Metabolismo en reposo (calorías) & 1419 & 191,8 & $1341(96,13)$ & $1694(191,48)$ \\
\hline Total, n (\%) & \multicolumn{2}{|c|}{63} & $49(77,7)$ & $14(22,3)$ \\
\hline
\end{tabular}

Tabla 2. Variables antropométricas finales y dosis máxima de liraglutida utilizada

\begin{tabular}{l|c|c|c|c|}
\multicolumn{1}{c|}{ Variable } & Media & DE & \multicolumn{2}{c}{ Mujeres } \\
\cline { 2 - 5 } & & & \multicolumn{2}{c}{ Media (DE) } \\
\hline Peso $(\mathrm{kg})$ & 72,7 & 12,36 & $68,6(7,56)$ & $87(15,0)$ \\
\hline IMC $\left(\mathrm{kg} / \mathrm{m}^{2}\right)$ & 27,6 & 3,19 & $26,9(2,64)$ & $29,9(3,92)$ \\
\hline Porcentaje de grasa corporal & 34,4 & 6,04 & $35,8(5,61)$ & $29,3(4,7)$ \\
\hline Dosis máxima de liraglutida $(\mathrm{mg})$ & 1,47 & 0,48 & $1,43(0,47)$ & $1,58(0,51)$ \\
\hline Total, $\mathrm{n}(\%)$ & & 63 & $49(77,7)$ & $14(22,3)$ \\
\hline
\end{tabular}


Tabla 3. Comparaciones variables antropométricas iniciales y finales

\begin{tabular}{l|c|c|c|c}
\hline \multicolumn{1}{c|}{ Variable } & Inicial (DE) & Final (DE) & $\begin{array}{c}\text { Porcentaje de } \\
\text { pérdida (DE) }\end{array}$ \\
\hline Peso $(\mathrm{kg})$ & $82,4(12,49)$ & $72,7(12,36)$ & 0,000 & $11,85(3,43)$ \\
\hline IMC $\left(\mathrm{kg} / \mathrm{m}^{2}\right)$ & $31,2(3,35)$ & $27,6(3,19)$ & 0,000 & $11,59(3,64)$ \\
\hline Porcentaje de grasa corporal & $41,06(5,3)$ & $34,4(6,04)$ & 0,000 & $11,67(6,96)$ \\
\hline Porcentaje de masa magra corporal & $27,02(5,4)$ & $26,4(5,91)$ & 0,235 & $2,25(4,11)$ \\
\hline Perímetro abdominal (cm) & $103(11,8)$ & $89,8(11,01)$ & 0,000 & $12,74(4,87)$ \\
\hline Metabolismo en reposo (calorías) & $1419(191,8)$ & $1,406(200,6)$ & 0,296 & $0,99(2,37)$ \\
\hline
\end{tabular}

la disminución de ese parámetro está estrechamente relacionada con la reducción del riesgo cardiovascular (8).

La reducción promedio de la adiposidad corporal fue del $11,67 \%$ y es llamativa la disminución estadísticamente no significativa de la masa magra, demostrada estadísticamente. No hay una clara explicación para este último resultado a pesar de ser un modelo de restricción calórica y rápida reducción de peso; sin embargo, se podría atribuir al modelo normoproteico con 1,2 g/kg día (9) y al mantenimiento de un plan de actividad física.

Otro aspecto para analizar es la tasa metabólica en reposo, la cual muestra una disminución que no es estadísticamente significativa, explicada quizás por el poco impacto en la masa muscular de los pacientes y no concuerda con la teoría que sustenta el riesgo de una nueva ganancia de peso basado en una disminución de la tasa metabólica en reposo (10), este aspecto podría ser importante al momento de lograr beneficios a largo plazo.

Existe preocupación por la recuperación del peso, un evento común en los métodos actuales de tratamiento de la obesidad $(11,12)$; sin embargo, algunos estudios han demostrado una persistencia de pérdida de peso superior al $10 \%$ del peso inicial después de un modelo VLCD al año de seguimiento (13). El modelo utilizado no reportó abandonos por eventos adversos o efectos secundarios.
El estudio tiene algunas limitaciones. Se trató de una fuente única de pacientes en la que se puede introducir algún sesgo de selección y diferencias iniciales. No se contó con un grupo de control, pues solo se describe de forma retrospectiva los resultados para un grupo de pacientes. Se requiere un seguimiento más extendido para evaluar la eficacia a largo plazo de la intervención.

Finalmente, consideramos que nuestros resultados deben confirmarse en cohortes más grandes para poder hacer recomendaciones generales a la población, pues se trató de un análisis retrospectivo, lo que limita la recolección e interpretación de muchos datos importantes.

\section{Conclusión}

Los resultados del presente estudio, centrado en un programa dietético con el uso de liraglutida, reemplazo de la cena y acompañamiento profesional 24/7, confirman los resultados en torno a ser una estrategia efectiva en la pérdida de peso a corto plazo en pacientes con obesidad. La reducción del peso ha demostrado ser lo suficientemente rápida durante el primer mes, lo que contribuye a garantizar la máxima adherencia; además, la disponibilidad de acompañamiento permanente y el control médico semanal pueden ser factores para tener en cuenta a la hora de abordar pacientes con obesidad y sobrepeso.

\section{Referencias}

1. GBD 2015 Obesity Collaborators, Afshin A, Forouzanfar MH, Reitsma MB, Sur P, Estep K, et al. Health Effects of Overweight and Obesity in 195 Countries over 25 Years. N Engl J Med. 2017;377(1):13-27.

2. Diabetes Prevention Program (DPP) Research Group. The Diabetes Prevention Program (DPP): description of lifestyle intervention. Diabetes Care. 2002;25(12):2165-71.

3. Sacks FM, Bray GA, Carey VJ, Smith SR, Ryan DH, Anton SD, et al. Comparison of weight-loss diets with different compositions of fat, protein, and carbohydrates. N Engl J Med. 2009;360(9):859-73.

4. Bray GA, Heisel WE, Afshin A, Jensen MD, Dietz WH, Long M, et al. The Science of Obesity Management: An Endocrine Society Scientific Statement. Endocr Rev. 2018;39(2):79-132.

5. Heymsfield SB, van Mierlo CA, van der Knaap HC, Heo M, Frier HI. Weight management using a meal replacement strategy: meta and pooling analysis from six studies. Int J Obes Relat Metab Disord. 2003;27(5):537-49.

6. Wadden TA, Hollander P, Klein S, Niswender K, Woo V, Hale PM, et al Weight maintenance and additional weight loss with liraglutide after low- 
calorie-diet-induced weight loss: the SCALE Maintenance randomized study. Int J Obes (Lond). 2013;37(11):1443-51.

7. The R Project for Statistical Computing [internet]. The R Foundation [citado el 20 de marzo de 2020]. Disponible en: https://www.r-project.org/

8. Pouliot MC, Després JP, Lemieux S, Moorjani S, Bouchard C, Tremblay A et al. Waist circumference and abdominal sagittal diameter: best simple anthropometric indexes of abdominal visceral adipose tissue accumulation and related cardiovascular risk in men and women. Am J Cardiol. 1994;73(7):460-8.

9. Paoli A, Rubini A, Volek JS, Grimaldi KA. Beyond weight loss: a review of the therapeutic uses of very-low-carbohydrate (ketogenic) diets. Eur J Clin Nutr. 2013;67(8):789-96.
10. Rosenbaum M, Hirsch J, Gallagher DA, Leibel RL. Long-term persistence of adaptive thermogenesis in subjects who have maintained a reduced body weight. Am J Clin Nutr. 2008;88(4):906-12.

11. Bueno NB, de Melo IS, de Oliveira SL, da Rocha Ataide T. Very-low-carbohydrate ketogenic diet v. low-fat diet for long-term weight loss: a metaanalysis of randomised controlled trials. Br J Nutr. 2013;110(7):1178-87.

12. Ebbeling CB, Swain JF, Feldman HA, Wong WW, Hachey DL, Garcia-Lago E et al. Effects of dietary composition on energy expenditure during weightloss maintenance. JAMA. 2012;307(24):2627-34.

13. Moreno B, Bellido D, Sajoux I, Goday A, Saavedra D, Crujeiras AB, et al Comparison of a very low-calorie-ketogenic diet with a standard lowcalorie diet in the treatment of obesity. Endocrine. 2014;47(3):793-805. 


\section{Medtronic}

\section{BOMBA DE INSULINA MINIMED ${ }^{\text {TM }} 670 \mathrm{G}$}

CON TECNOLOGÍA SMARTGUARD ${ }^{\text {TM }}$

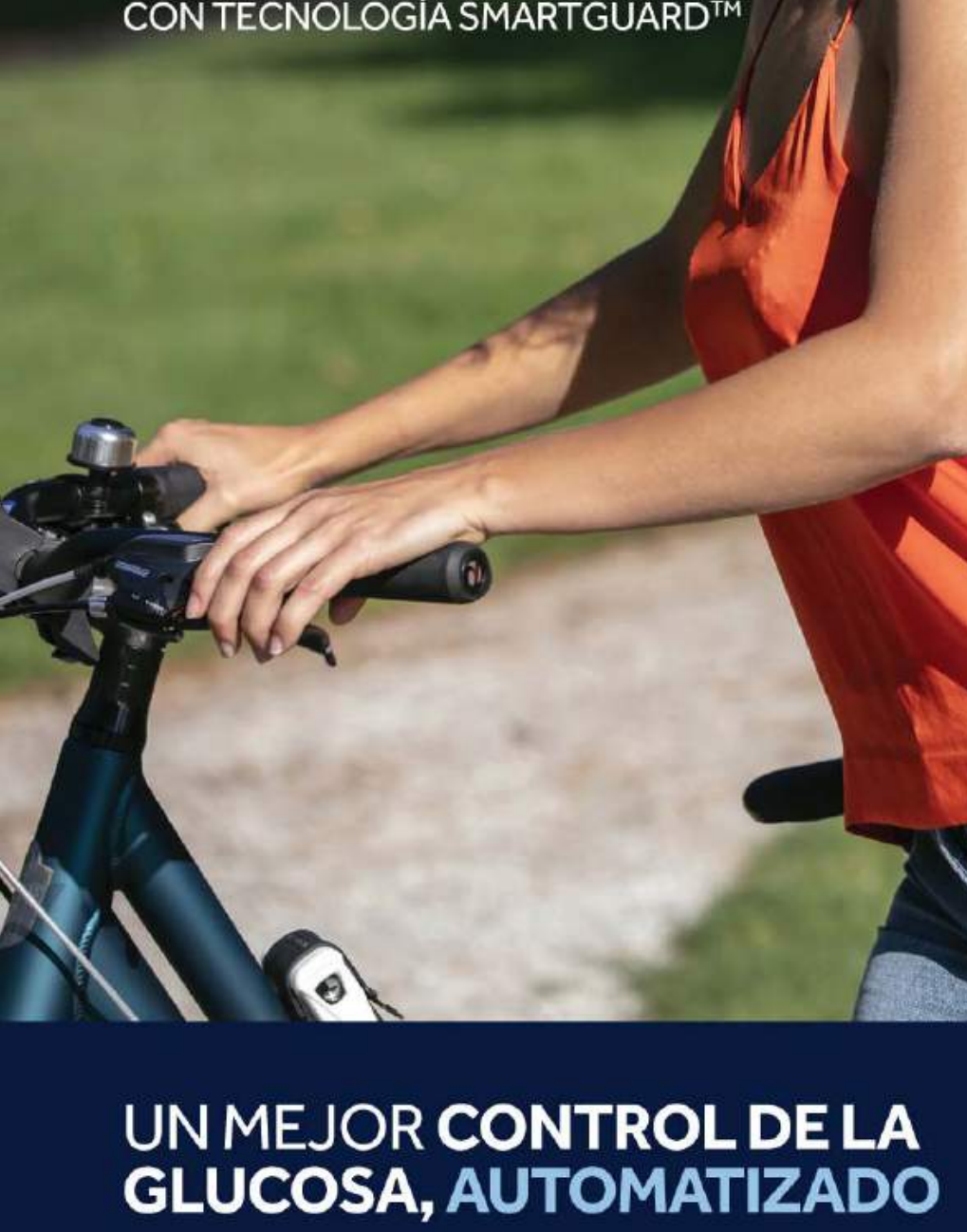

MÁS TIEMPO ENRANGO

Al automatizar la entrega de insulina, el sistema MiniMed $^{\top M} 670 \mathrm{G}$ con tecnología SmartGuard, ayuda a incrementar el tiempo en rango del paciente $^{1.2}$

\section{MEJOR CONTROL}

¿Cómo sería su día a día si los valores de glucosa de sus pacientes se mantuvieran entre 70 y $180 \mathrm{mg} /$ dL de dia y de noche, automáticamente?*

¡Ahora es posible!

\section{RESULTADOS} CLIINICOS ${ }^{1,2}$

- Reducciónen HbA1c de 7,4 a 6,9

- Incremento en TIR de $66,7 \%$ a $72,2 \%$

- Cero casos de hipoglucemia grave**

\section{CARELINK SYSTEM}

Gestione y realice un seguimiento del tratamiento con bomba de insulina de sus pacientes, a través del software en linea que genera informes completose intuitivos.
Ajusta automáticamente la infusión de insulina basal según las lecturas de monitorización continua de glucosa (MCG), adaptándose a las necesidades únicas de insulina de cada paciente.* 